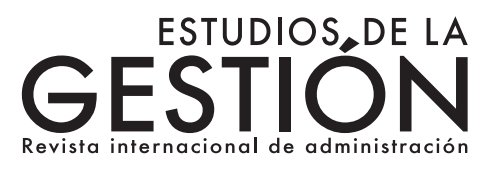

\title{
Industria 4.0: el reto en la ruta hacia las organizaciones digitales
}

Industry 4.0: The Challenge on the Road to Digital Organizations

Indústria 4.0: o desafio no caminho às organizações digitais

\author{
Dorian Mora-Sánchez \\ Universidad UTE. Quito, Ecuador \\ dorian.mora@gmail.com \\ https://orcid.org/0000-0002-9786-3878 \\ Luis Guerrero-Marín \\ Universidad UTE. Quito, Ecuador \\ luisguerreromarin@gmail.com \\ https://orcid.org/0000-0003-2797-5871
}

DOI: https://doi.org/10.32719/25506641.2020.8.7

Recibido: 15 de febrero de 2020 - Revisado: 6 de marzo de 2020

Aceptado: 18 de abril de 2020

Artículo de investigación

Licencia Creative Commons

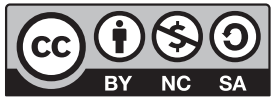




\section{Resumen}

La cuarta revolución industrial, conocida como Industria 4.0, promueve que los sistemas físicos industriales estén conectados al mundo digital, forzando que las organizaciones salgan de su statu quo. Esta realidad representa un antecedente para que las organizaciones conciban esta nueva revolución para poder migrar a este paradigma desde donde se encuentren actualmente. El objetivo principal de este artículo es conocer los retos y desafíos que trae consigo la cuarta revolución industrial para las organizaciones, a través de exteriorizar su importancia y características, y explicando su influencia en aspectos relacionados a su implementación en las organizaciones. La metodología exploratoria empleada incluye una revisión de literatura en las bases de datos científicas más importantes, en aspectos específicos como: las revoluciones industriales, rasgos característicos de la Industria 4.0, y su influencia en las organizaciones. En los resultados se puede evidenciar el influjo de las distintas revoluciones industriales, los detalles intrínsecos de la Industria 4.0, la atención que puede requerir su implementación dentro de las organizaciones, y abrir la posibilidad de tener un enfoque de organización 4.0 sostenible. Finalmente, se concluye que para migrar a la Industria 4.0, una organización debe entender los retos en la gestión de sus operaciones, del talento humano, energética, operativa, estratégica y de sostenibilidad.

Palabras clave: Cuarta revolución, retos, Industria 4.0, organización, digitalización.

JEL: O33 Cambio tecnológico: opciones y consecuencias; difusión.

\section{Summary}

The fourth industrial revolution, known as Industry 4.0, encourages industrial physical systems to be connected to the digital world, forcing organizations out of their Status Quo. This reality represents a precedent for organizations to conceive this new revolution in order to migrate to this paradigm from where they currently are. The main objective of this article is to know the challenges that the fourth industrial revolution brings for organizations, by externalizing its importance and characteristics, and explaining its influence on aspects related to its implementation in organizations. The exploratory methodology used includes a literature review in the most important scientific databases on specific aspects such as: industrial revolutions, characteristic features of Industry 4.0, and its influence on organizations. The results can show the influence of the different industrial revolutions, the intrinsic details of Industry 4.0, the attention that its implementation may require within organizations, and open up the possibility of having a sustainable 4.0 organization approach. Finally, it is concluded that in order to migrate to Industry 4.0, an organization must understand the challenges in managing its operations, human talent, energy, operations, strategy, and sustainability.

Keywords: Fourth Revolution, challenges, industry 4.0, organization, digitization.

JEL: O33 Technological change: options and consequences; diffusion. 


\section{Resumo}

A quarta revolução industrial, conhecida como Indústria 4.0, promove que os sistemas físicos industriais estejam conectados ao mundo digital, forçando as organizações a saírem de seu Status Quo. Essa realidade representa um antecedente para que as organizações concebam essa nova revolução de forma a poder migrar a esse paradigma a partir do lugar onde se encontram atualmente. O objetivo principal deste artigo é conhecer os desafios que a quarta revolução industrial traz para as organizações. Exteriorizaremos sua importância e suas características, explicando sua influência em aspectos relacionados à sua implementação nas organizações. A metodologia exploratória empregada inclui uma revisão da literatura nas bases de dados científicas mais importantes no que diz respeito a aspectos específicos, tais como: as revoluções industriais, as características da Indústria 4.0 e sua influência nas organizações. Os resultados evidenciam a influência das distintas revoluções industriais, os detalhes intrínsecos da Indústria 4.0 e a atenção que pode requerer sua implementação dentro das organizações, abrindo-se assim a possibilidade para um enfoque sustentável de organização 4.0. Finalmente, conclui-se que, para migrar à Indústria 4.0, uma organização deve entender os desafios da gestão de operações, talentos, energia, estratégia e sustentabilidade.

Palavras-chave: Quarta revolução, desafios, indústria 4.0, organização, digitalização. JEL: O33 Mudança tecnológica: escolhas e consequências; processos de difusão.

\section{Introducción y estado de la cuestión}

través de generaciones, y de las distintas revoluciones industriales, las organizaciones se han convertido en un sector esencial e inteligente en la sociedad debido a que se han incorporado nuevas tecnologías, sistemas de gestión de energía, automatización de producción y de procesos administrativos, entre otros, los cuales les han permitido mejorar los procesos empresariales, no solo desde el punto de vista del tiempo, sino también desde la calidad.

La última de estas revoluciones, conocida también como industria 4.0 (I4.0), congrega un sinnúmero de tecnologías ya establecidas y en desarrollo para generar una revolución a gran escala capaz de cambiar la forma de operar a las organizaciones, incluyendo los conceptos de ética en este nuevo paradigma (Calvo 2017). Pero las organizaciones encuentran distintos desafíos que deben tomar en cuenta al momento de buscar la transformación digital.

Esta problemática genera la interrogante: ¿cuáles son los nuevos retos y desafíos de las organizaciones con la llegada de I4.0? Por esta razón, el 
objetivo principal de este artículo es conocer los retos y desafíos que trae consigo la cuarta revolución industrial para las organizaciones, cuyos objetivos específicos se enfocan en presentar la importancia y las características de la I4.0 y de explicar su influencia en muchos aspectos relacionados a su implementación en las organizaciones.

La transformación digital representa un desarrollo adicional dentro de la organización, incluyendo la gestión de los procesos llevados a cabo a través de la automatización, la robótica y el intercambio de datos (Fernández y Pajares 2016), permitiendo integrar nuevos recursos dentro de la organización (Bartodziej 2017).

El hecho de que la industria pueda fabricar productos altamente personalizados e inteligentes ayudados por tecnologías como big data (BD), computación en la nube, inteligencia artificial (AI), internet de las cosas (IoT), internet industrial de las cosas (IIoT), manufactura aditiva (3D-P), redes 5G, realidad virtual (VR), realidad aumentada (AR), ciberseguridad, blockchain, entre otras, está reforzada en la congregación de las mencionadas tecnologías para dar paso a una nueva revolución industrial.

Este artículo va más allá del estado del arte, en donde ya se presentan algunos retos y riesgos relacionados a la llegada de la I4.0, especialmente con orientación a la responsabilidad social de las organizaciones (Mora 2019a; 2019b); en todo el documento se hace referencia al estado del arte de las tecnologías de la I4.0, para avanzar y enfocarse a los ámbitos que las organizaciones tendrán que prestar atención para una posible transformación digital.

\section{Material y métodos}

La metodología utilizada es una investigación exploratoria, en la que se busca realizar un primer contacto con el paradigma llamado I4.0 y su relación con las organizaciones, conocer y familiarizarse con este tema de estudio aún desconocido, y así lograr tener un horizonte superficial que permita ofrecer una primera aproximación a la realidad actual y de lo que les espera a las organizaciones. La exploración responde a una contribución sobre los desarrollos recientes en el ámbito de la cuarta revolución industrial y 
su impacto en las organizaciones, teniendo en cuenta sus beneficios, riesgos y desafíos a los que se enfrentan.

Este estudio se centró en la pesquisa de bibliografía en temas relacionados con las revoluciones industriales a través de los tiempos, las peculiaridades propias de la I4.0 como la automatización industrial (Gil 2017).

Para la recopilación se utilizó como criterio de búsqueda las palabras clave del artículo: cuarta revolución, reto, industria 4.0, organización, digitalización. La recopilación de literatura hizo sobre la base de una gran colección de artículos, libros e informes. Se limitó a bibliografía en los idiomas inglés, español y alemán, y se ejecutó buscando en tres fuentes diferentes: 1. repositorios científicos como JCR, Scopus, Springer, Taylor \& Francis, Gale, Redib, Refseek, Scielo, Dialnet Métrics, Academia.edu, Redalcyc, Re.public@polimi, archivos HAL, Sage journal, J-Stage e IEEE Xplore; 2. se consideraron algunos libros de última generación para explorar los últimos conceptos relacionados; y 3. informes especiales de organizaciones globales relacionadas a la I4.0. La evaluación y selección de la literatura se basó en criterios de relevancia a la pregunta de investigación y calidad de las publicaciones, y luego de este filtro se obtuvo la literatura referenciada y citada en este artículo. La información se elaboró finalmente con el enfoque en el problema de investigación, información existente, conclusiones y resultados encontrados en la literatura.

El estudio de la I4.0 arranca desde sus faces históricas, descripción, interiorización, hasta las implicaciones de una virtual implementación y todos los aspectos a tener en cuenta en las organizaciones.

\section{Análisis de la delineación histórica}

Al referirse al término revolución industrial, se enfatizan las innovaciones tecnológicas que comenzaron a reemplazar la capacidad humana con maquinaria, así como la fuerza humana y animal con las basadas en nuevas energías, y dando lugar a diferentes ciclos económicos (Basco et al. 2018; Humphries y Schneider 2018). En la figura 1 se representa un breve resumen de las diferentes revoluciones industriales y sus semblantes característicos. 
Figura 1

Desarrollo de las revoluciones industriales

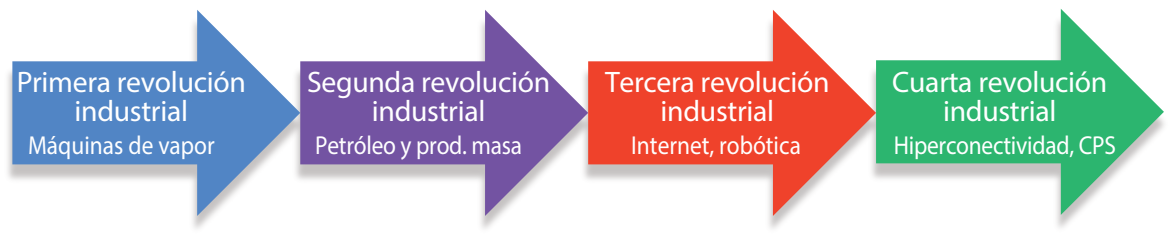

Elaboración propia.

\section{Primera revolución industrial}

Este período se desarrolló en Gran Bretaña desde mediados del siglo XVIII hasta el XIX. La particularidad general se fundamentó en sociedades agrícolas y rurales que crecieron y se transformaron en actividades industriales y urbanas, respectivamente (Crafts 1996).

Las industrias del hierro y textil se favorecieron especialmente de un desarrollo ingenioso llamado máquina de vapor, que fue el punto de arranque del desarrollo de los motores que la sociedad conoce actualmente, y que proporcionó múltiples usos en la industria (Mokyr 1999).

\section{Segunda revolución industrial}

La segunda revolución industrial se llevó a cabo entre 1870 y 1914, poco antes de la Primera Guerra Mundial, período en el que se evidenció un gran crecimiento industrial que generó la expansión y la creación de organizaciones (Mokyr 1998).

Entre las nuevas materias primas destacan el acero, y el petróleo y sus respectivos productos derivados; pero no solo los recursos fueron los avances más importantes. El sector energético también se vio beneficiado con la electricidad. 


\section{Tercera revolución industrial}

Este período, que inició en los años ochenta y aún continúa, se fundamenta en la automatización y la tecnología digital. Con el advenimiento del internet, sistemas informáticos y comunicaciones, las organizaciones pudieron generar diferentes dispositivos electrónicos que son de uso general (Mowery 2009).

Este período se ha caracterizado por enfocarse en una sociedad de la información, sumado a la internet, la automatización y la robotización.

\section{Cuarta revolución industrial}

Los períodos anteriores han dejado muchas contribuciones a la sociedad y a las organizaciones, y es aquí en donde surge la astucia de integrar nuevas tecnologías en un cuarto período industrial (Del Val 2016). Y como un agregado, a pesar de las contribuciones que generaron las anteriores revoluciones, hoy se cuestiona el impacto ambiental y social que han generado en la historia y que aún lo siguen haciendo (Yin, Stecke y Li 2018).

La hiperconectividad hace referencia a que todos los dispositivos participarían y colaborarían entre sí, generando nuevas capacidades en los sistemas. Los sistemas de sistemas (SoS), también conocidos como supersistemas o megasistemas (Mora, Taisch y Colombo 2012a; Mora et al. 2012) dieron la primicia a sistemas hiperconectados en implementaciones de automatización industrial sostenibles (Mora, Taisch y Colombo 2012b).

I4.0 apareció inicialmente como un término en 2010, y un año después se lo presentó en la Hannover Messe en Hannover, Alemania. Este país, conocido como uno de los fundadores de la I4.0, lo incluyó en su agenda gubernamental como un proyecto clave en estrategias tecnológicas, ineludible para aumentar su PIB y estimular la revolución digital (Schwab 2017).

I4.0 es compatible con varias tecnologías, tanto ya establecidas como nuevas y en desarrollo (Del Val 2016). Muchas de las tecnologías integradas en la I4.0 facilitan a las organizaciones desarrollar una producción inteligente y personalizada, permitiendo la transición a nuevos sistemas ciberfísicos 
(CFS) que conectan un organización con su gemelo digital (Telukdarie et al. 2018).

La I4.0 es un paradigma que podría fomentar el espíritu empresarial en todo el mundo (Hidayat y Yunus 2019). Hay muchas pesquisas sobre I4.0, sus tecnologías, y los bosquejos de organización inteligente (Thoben, Wiesner y Wuest 2017).

\section{Análisis de los rasgos propios de la 14.0}

Cada día se pueden evidenciar mayores interacciones entre máquinas y humanos gracias al desarrollo de nuevas tecnologías (Frank, Dalenogare y Ayala 2019). En relación con la I4.0, dichas tecnologías son integradas desde una perspectiva de interoperabilidad y, aunque al día de hoy aún no existe un consenso sobre las tecnologías integradas a manera de orquesta en la I4.0, las más frecuentes encontradas en la literatura se exhiben en este artículo (figura 2).

\section{Figura 2 \\ Orquestación de la 14.0}

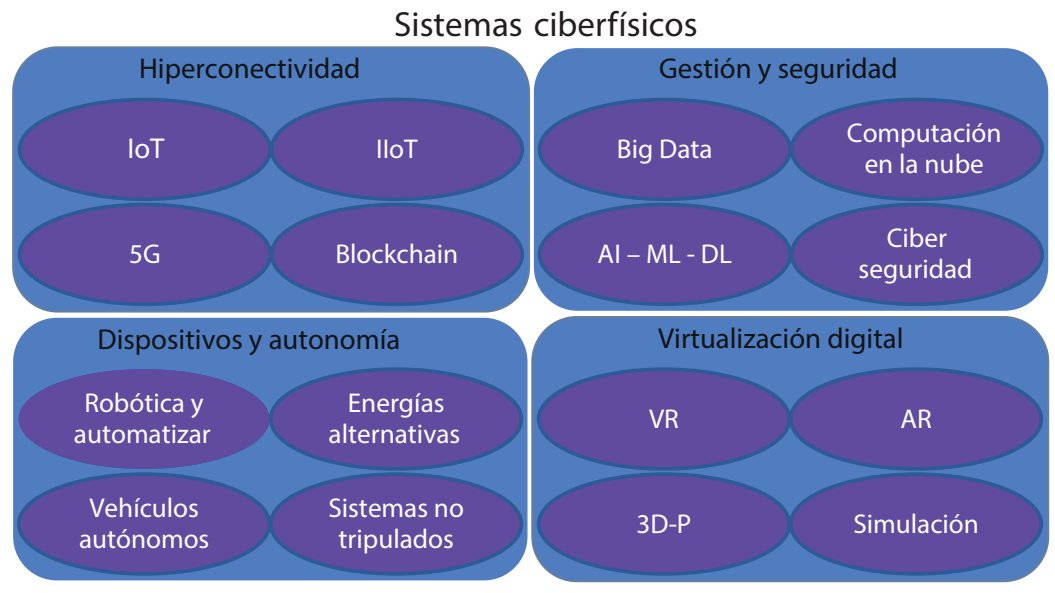

Fuente y elaboración propia. 


\section{Sistemas ciberfísicos (CPS)}

La característica esencial de estos sistemas son los componentes físicos y digitales (informáticos, comunicacionales, de almacenamiento), los cuales están intensamente entretejidos con un mundo físico, y con el valor agregado de comunicarse entre sí (Shrouf, Ordieres y Miragliotta 2014).

Una de las aplicaciones más llamativas de los CPS es la logística 4.0 (Neumann 2019), en donde estos sistemas tanto físicos como digitales son muy útiles para monitorear productos y equipos proporcionando variables físicas como temperatura, humedad, ubicación, cantidad, etc. (Wang, Törngren y Onori 2015; Wang, Wan, Li et al. 2016; Wang, Wan, Zhang et al. 2016).

\section{Hiperconectividad}

La hiperconectividad busca la sincronización de todos los actores en un CPS (Coyle et al. 2018). Cuando se trata de conectividad dentro del contexto de la I4.0, esa percepción se relaciona al IoT, y en el ámbito industrial a su extensión llamada IIoT. La hiperconectividad de todos los actores IoT e IIoT y sus procesos agregó velocidad, y los datos generaron un análisis automático, inteligente, rápido y preciso en toda la cadena (Ananth, Seshadri y Vasher 2009).

IoT se basa principalmente en dispositivos como sensores, actuadores y controladores, identificados y conectados a dicha súper red (Cueva, Rodríguez y Montenegro 2015; Barrio 2018). Las aplicaciones de IoT cubren prácticamente todos los sectores, incluidos el de la industria, de servicios y productos, de gestión ambiental, de ciudades inteligentes, etc. (Kagermann, Wahlster y Helbig 2013; Mattern 2013).

IIoT, en el mismo contexto, es una versión de IoT basada en aplicaciones puramente industriales. El concepto de IIoT se refiere al uso de tecnologías de IoT en procesos industriales, que integra capacidades como procesamiento, almacenamiento y comunicación para controlar uno o más procesos físicos (Branger y Pang 2015).

Otro aspecto indispensable en la hiperconectividad son las redes de comunicaciones, y en la actualidad no se espera menos que una red de conec- 
tividad de quinta generación $(5 \mathrm{G})$. Estas redes móviles son una verdadera revolución y más aún si se las enfoca dentro de una solución de I4.0 (Xu et al. 2018). Estas redes inteligentes de comunicaciones de banda ancha móvil pueden ofrecer velocidades cien veces más altas que las de cuarta generación actuales y resolverían el problema causado por la gran explosión de la demanda de comunicaciones, servicios y aplicaciones móviles (Bangerter et al. 2014; Talwar et al. 2014).

Así mismo, la tecnología blockchain implica un paso más en la hiperconectividad, ya que significa descentralización y validación de información multimodal, convirtiéndose así en una tecnología clave en esta I4.0 (Apte y Petrovsky 2016; Bahga y Madisetti 2016).

\section{Gestión y seguridad de la información}

La masiva concepción de datos debe analizarse muy cuidadosamente para encontrar patrones, tendencias y oportunidades para mejorar y optimizar los procesos y operaciones (Malvicino y Yoguel 2016), a través de procedimientos llamados analítica de datos. Estos datos pueden procesarse $\mathrm{y}$ analizarse de tal manera que se podrían extraer patrones, que pueden ser utilizados por otras aplicaciones y servicios como, por ejemplo: sistemas de salud, sistemas de información pública, gestión de la ciudad, eficiencia energética, gestión de residuos, gestión del agua, entre otros (Ries y Gins 2017).

La gran cantidad de datos pueden ser procesados y almacenados en servidores locales, pero la I4.0 se enfoca al uso de la nube. Esta tecnología facilita la gestión de la información y su almacenamiento en la misma red al evitar que las organizaciones adquieran su propia infraestructura física (Murazzo et al. 2013). El beneficio para la organización es tener la información correctamente almacenada y procesada desde cualquier ubicación (Vachálek et al. 2017).

Por otro lado, dicho gran volumen de datos generados ha permitido la investigación y el desarrollo de sistemas de AI capaces de procesar todos esos datos de manera confiable y esencialmente de forma autónoma. Esta tecnología está actualmente en uso y es compatible con otras tecnologías 
como aprendizaje de máquina (ML) y aprendizaje profundo (DL) (Dopico et al. 2016).

Finalmente, otro aspecto muy necesario al gestionar información es su seguridad. La ciberseguridad se alinea en tener sistemas de información y comunicación seguros y más confiables, y que estos sean en lo posible lo menos vulnerables para lograr salvaguardar los datos (Sancho 2017).

\section{Dispositivos y autonomía}

Luego de la tercera revolución aparece la automatización industrial, la cual permite el desarrollo de procesos independientemente controlados, que logran sistemas dinámicos (Serna, Catalán y Blesa 2015; Kolberg y Zühlke 2015). Pero aún existen muchísimas organizaciones que mantienen sus procesos manuales y no pasan de la segunda revolución industrial, lo cual hace más difícil la migración a la cuarta revolución.

La robótica también se empodera como aporte a la I4.0, especialmente en procesos repetitivos e interconectados, como por ejemplo tareas de empaque y etiquetado (Ortega Moody et al. 2016). Además, los vehículos autónomos dentro de la I4.0, debido a que estos dispositivos pudieran transportar mercancías de una estación a otra, sin la intervención humana (Pieroni, Scarpato y Brilli 2018).

Otra tecnología disruptiva dentro de una solución de I4.0 es la manufactura aditiva, que va más allá del sistema tradicional de abastecimiento de productos para llegar a permitir la fabricación de piezas complejas en un tiempo récord, a un costo competitivo a través de la utilización de impresoras 3D (3D-P) (Thompson et al. 2016; Dilberoglu et al. 2017).

Adicionalmente, y actuando de manera transversal, se encuentran las nuevas tecnologías energéticas, las cuales permiten obtener eficiencia energética y el uso de energías alternativas (Mora 2019a).

Finalmente, la I4.0 no duda en la utilización de sistemas no tripulados, como es el caso de los drones conectados dentro de este mundo ciberfísico, permitiendo así interconectarse e interactuar con las diferentes unidades de proceso en las organizaciones (Mora 2019a). 


\section{Virtualización y digitalización}

La AR se encarga de generar una capa virtual por sobre la real, y utiliza el entorno físico real para proporcionar datos e información en tiempo real, ejemplificando procesos como el almacenamiento y el transporte, como la recepción, la ubicación, la recolección, el embalaje, la carga del vehículo y la entrega final (Paelke 2014).

Por otro lado, la VR está más relacionada con un mundo nuevo, donde el cerebro está conectado y el personal podría pensar que el mundo virtual es, de hecho, el real (Kovar et al. 2016).

Dentro de la I4.0 también se hace referencia a la simulación 2D y 3D, las cuales se basan, en el caso de procesos industriales logísticos, en la planificación y el estudio de escenarios operativos que permitan a los gerentes de la cadena de suministro y a los gerentes de procesos logísticos conocer de antemano y visualmente el comportamiento del flujo de materiales dentro y fuera de la empresa.

\section{Resultados: 14.0 y su influencia en la organización}

El arribo de la I4.0 genera nuevos retos y desafíos en las organizaciones, razón por la cual es importante tener una visión general de las áreas más importantes en que las empresas tendrán que reajustarse y restructurarse, que se encuentran concisos en la figura 3 .

\section{0 y la gestión de proyecto 14.0 (P-14.0)}

La implementación de las tecnologías que integran la I4.0 y que se adaptan a las necesidades de la organización tendrá que ser analizada muy a detalle e involucra una gestión muy dedicada para el éxito (Moeuf et al. 2018).

Primeramente, se tendrían que desarrollar los distintos estudios necesarios, los cuales, dependiendo de su realidad, podrían incluir: prefactibilidad, factibilidad, tamaño, ubicación, ingeniería, organización, programa, tecnolo- 
Figura 3

\section{La 14.0 en coherencia a las organizaciones}

\section{Organización}

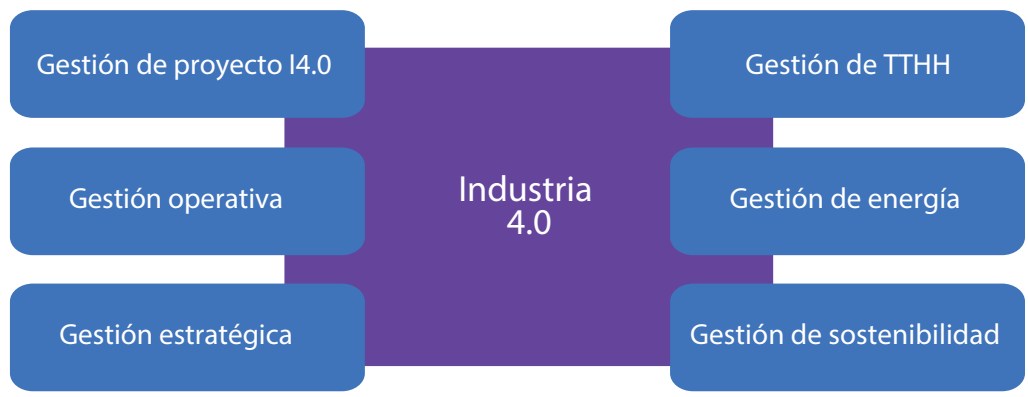

Elaboración propia.

gía, economía y finanzas, y se agregarían redes, manejo de datos, infraestructura digital, infraestructura de ciberseguridad, entre otras. Luego, basados en una evaluación en detalle, se tomaría la decisión de si es conveniente o no la implementación de dicho P-I4.0. Finalmente, en caso de una respuesta previa positiva, se procedería a la ejecución e implementación de dicho proyecto. A continuación, se tendrían que tomar en cuenta los aspectos relacionados a la (auto)operación del mismo (Müller, Buliga y Voigt 2018).

\section{0 y la gestión operativa}

La hiperconectividad permitirían el control de la producción en tiempo real, lo que lleva a una mejora en la calidad de cada uno de los procesos involucrados y aumentaría la productividad de las máquinas y las líneas de producción (Ramachandran et al. 2015).

El desarrollo continuo de la I4.0 permitiría tomar acciones predictivas y optimizar todos los elementos que influyen en las empresas, lo que permitiría que las fábricas se vuelvan inteligentes, flexibles, (semi)autónomas, y con minúsculos defectos (Rodič 2017). 


\section{0 y la gestión estratégica}

La I4.0 promueve que las organizaciones primeramente desarrollen un análisis circunstancial para poder identificar cuáles serían las nuevas fortalezas, oportunidades, debilidades y amenazas al migrar dicha organización a la cuarta revolución industrial, y compararlo con la línea base actual de la organización.

Esto permitirá definir cuáles serían los nuevos objetivos y resultados esperados, los tiempos para lograrlo y las políticas restauradas de la organización, para poder definir el grupo de estrategias así como los recursos tecnológicos y digitales necesarios para lograr el objetivo (Mora 2019b).

\section{0 y la gestión de talento humano (TTHH)}

Uno de los grandes desafíos de I4.0 son los perfiles profesionales que esta nueva revolución empresarial exige. Esta revolución implicará aumentar el personal en áreas como almacenamiento de datos, gestión e interpretación de datos, seguridad cibernética, monitoreo de hiperconectividad, gestión de red, computación en la nube, robótica, programación, entre otras; pero también existirán nuevos puestos de trabajo requeridos que tendrán que ser creados, como limpiador de datos, administrador de interacciones, agente de algoritmos, agente de AI, agente de minería de datos, dependiendo de las exigencias y del tipo de organización. Además, se requerirá expertos en gestión que establezcan una relación óptima entre el hombre y la máquina, que coexistirían en todo el proceso de producción (Zhou et al. 2018).

\section{0 y la gestión energética}

La implementación de I4.0 implicará un incremento significativo en el consumo de energía, debido al incremento de demanda por cada dispositivo que sea instalado dentro de la red de hiperconectividad. Y en este contexto, las nuevas tecnologías energéticas permitirán responder a la nueva demanda de energía para migrar a la I4.0 (Huang et al. 2017). Tanto los sistemas com- 
putacionales y de almacenamiento de datos, sistemas de comunicación, los sistemas de análisis de datos y toda la tecnología dura utilizada para la transformación y el movimiento de materiales y personas, requerirán energía para su operación. Algunas de las energías alternativas vistas desde una perspectiva de I4.0 se pueden resumir en: energía solar, energía eólica, energía mareomotriz, biomasa, etc. (Rodríguez 2008; IPCC 2011; Salhaoui et al. 2018).

\section{0 y la gestión de sostenibilidad}

La I4.0 en la actualidad, como facilitador del desarrollo tecnológico, ha contribuido al surgimiento de la era de la información y los datos. Ahora, la cantidad de datos generados en todos los aspectos de la vida diaria es grande, y con una perspectiva ascendente para el futuro (Stock et al. 2018). Esto implica la responsabilidad de las organizaciones con respecto a la gestión de dichos datos, y a que estas nuevas manipulaciones no representen impactos negativos económicos, sociales o ambientales, y por el contrario, se aporte con los objetivos de desarrollo sostenible (Van der Velden 2018).

La cuarta revolución industrial trae consigo muchos beneficios, pero es importante entender si las sociedades en donde se implementarán están listas para su adaptación, y si la empresa entiende y reflexiona que la responsabilidad social empresarial (RSE) debe ser un pilar al momento de migrar a la I4.0 (González 2016).

\section{Una visión de organización 4.0 sostenible (04.0S)}

Finalmente, la I4.0 representa un reto para las organizaciones respecto a la sostenibilidad y continuo mejoramiento, ya que exige un proceso sistemático de seguimiento continuo y evaluación permanente de los resultados frente a metas y planes propuestos, proceso que debería ser recurrente en períodos definidos por las mismas compañías. Este enfoque de sistema de gestión de sostenibilidad de la I4.0 podría ser visto como una oportunidad para las organizaciones. 
Figura 4

Enfoque de organización 4.0 sostenible

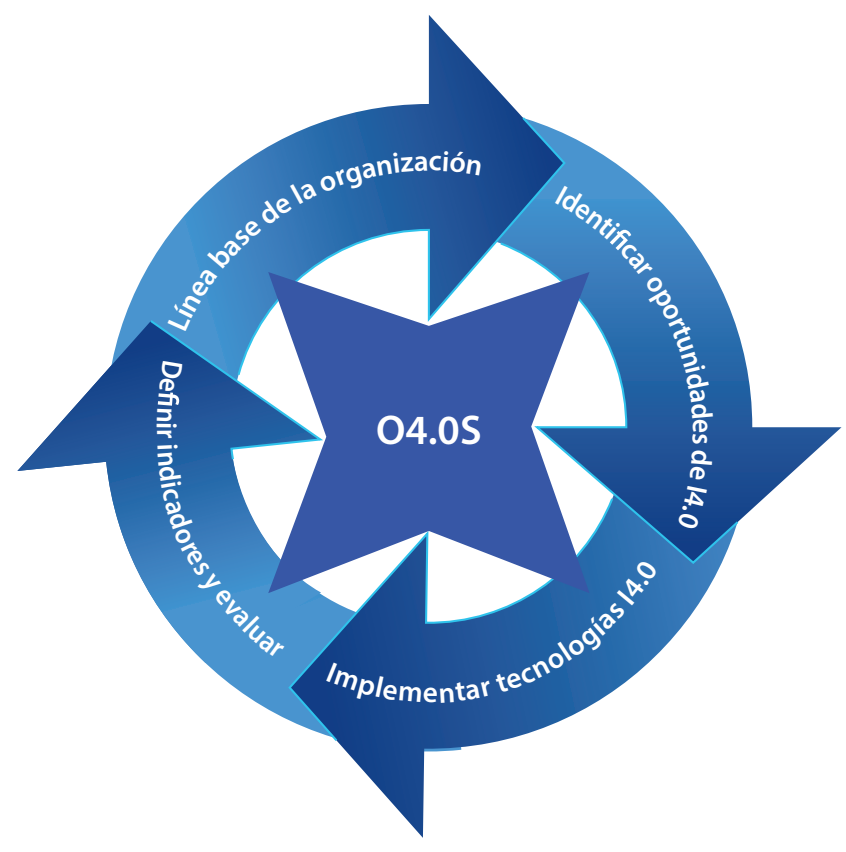

Fuente y elaboración propia.

\section{Línea base de la organización}

En esta primera fase se propone un esquema de diagnóstico-intervención que comprende el reconocimiento de una situación inicial de la compañía respecto a los parámetros I4.0, el reconocimiento de oportunidades de I4.0 aplicables a la organización.

\section{Identificar oportunidades de 14.0}

La segunda fase involucraría el reconocimiento de oportunidades de I4.0 aplicables a la organización. 


\section{Implementar tecnologías 14.0}

La tercera fase representa una etapa de implementación de proyectos que den respuesta al aprovechamiento de las oportunidades identificadas. Dichos proyectos tecnológicos involucran un portafolio de proyecto con una respectiva hoja de ruta, tomando en cuenta las realidades y la línea base de cada organización.

\section{Definir indicadores y evaluar}

Finalmente, la fase final sería la determinación de indicadores de sostenibilidad deseados, y una etapa de evaluación de resultados de los proyectos y cambios implementados, siendo esta etapa final el origen de un nuevo ciclo de diagnóstico e intervención para así generar un proceso cíclico sostenible de seguimiento, evaluación y mejoramiento que lleve a la organización a ser una O4.0S.

Este artículo finaliza con la propuesta de una futura O4.0S (figura 4) que deberá ser investigada posteriormente desde la academia, y así abrir las puertas a un nuevo aporte para las organizaciones.

\section{Discusión}

La I4.0 es una revolución industrial que implica una toma de decisiones por parte de las organizaciones con el propósito de migrar desde la segunda o tercera revolución industrial y encarrilarse en el tren de la cuarta revolución industrial.

El estudio muestra que son muchos los retos, desde distintos ámbitos de acción, para las organizaciones que deseen migrar hacia la cuarta revolución industrial. Inicialmente, tendrán que analizar su línea base y darse cuenta en qué revolución se encuentran y si están aptas para dar el salto. Las diferencias financieras, económicas, ambientales, sociales, operacionales, tecnológicas, estratégicas y de toda índole en las organizaciones llevan a una situación en que una posible migración hacia la I4.0 se tenga que analizar detenidamente, 
ya que inclusive en países en vías de desarrollo existen muchas empresas en donde sus modelos industriales de gestión y de operación se encuentran aún en la segunda revolución industrial. Sería importante que los países en vías de desarrollo generen políticas de desarrollo productivo alineadas con la I4.0, y de esta manera las organizaciones tengan mayores facilidades para una digitalización industrial.

Los resultados presentan un panorama adaptable desde el punto de vista de la realidad de los países industrializados, pero se ve más complicado para las organizaciones de los países en vías de desarrollo. Los desafíos de las organizaciones para poder migrar no solo tendrán que enfocarse en su línea base, sino también en la gestión netamente energética, operativa y estratégica de talento humano y de sostenibilidad.

Para fines de una futura investigación se recomienda proponer una hoja de ruta para la implementación de I4.0, y estudiar la transición de una compañía contemporánea a una $\mathrm{O} 4.0 \mathrm{~S}$.

\section{Conclusiones}

Con base en los resultados presentados en este artículo, se presentan los distintos retos que posiblemente enfrentarán las organizaciones que tengan como objetivo transformarse digitalmente. Se pudo concluir que los desafíos para las organizaciones en términos de digitalización industrial están muy relacionados a las características propias de la I4.0, especialmente en lo que se refiere a la misma factibilidad de su implementación, de sus operaciones, del talento humano, de su gestión energética, operativa, estratégica y de sostenibilidad.

La integración de tecnologías I4.0 en una organización permitirá que la estrategia de empresa se actualice dentro de los nuevos conceptos 4.0, que sus operaciones de producción más eficientes y personalizadas se desarrollen de una manera más limpia y sostenible a través de la utilización de energías alternativas y con estrategias implementadas de gestión eficiente de la energía, y que el talento humano tenga que ser formado de acuerdo con la nueva realidad 4.0. En mayor detalle, las nuevas oportunidades relacionadas a las nuevas tecnologías I4.0 permiten a las organizaciones evolucionar hacia 
nuevas organizaciones 4.0. Estas coyunturas pueden ser resumidas como: personalización de productos y servicios, control y detección temprana de problemas, consumo responsable de recursos como materias primas, insumos, energía, etc., reducción en la generación de residuos, decisiones como resultado de análisis de datos, satisfacción del cliente y mejora de la imagen, producción eficiente y competitiva, consumo eficiente de energía, control y monitoreo de la demanda de energía, y colateralmente contribución al desarrollo sostenible del planeta.

\section{Referencias}

Ananth, Iyer, Sridhar Seshadri y Roy Vasher. 2009. Administración de la cadena de suministro TOYOTA. Un enfoque estratégico a los principios del célebre sistema de TOYOTA. Ciudad de México: McGraw-Hill.

Apte, Shireesh, y Nikolai Petrovsky. 2016. "Will Blockchain Technology Revolutionize Excipient Supply Chain Management?". Journal of Excipients and Food Chemicals 7 (3): 76-78. https://bit.ly/2Bufjxy.

Bahga, Arshdeep, y Vijay Madisetti. 2016. "Blockchain Platform for Industrial Internet of Things". Journal of Software Engineering and Applications 9 (10): 533-346. https://doi. org/10.4236/jsea.2016.910036.

Bangerter, Boyd, Shilpa Talwar, Reza Arefi y Ken Stewart. 2014. "Networks and Devices for the 5G Era". IEEE Communications Magazine 52 (2): 90-96. https://doi.org/10.1109/ MCOM.2014.6736748.

Barrio Andrés, Moisés. 2018. Internet de las cosas. Madrid: Reus.

Bartodziej, Christoph Jan. 2017. The Concept Industry 4.0: An Empirical Analysis of Technologies and Applications in Production Logistics. Berlin: Springer. https://oi.org/10. 1007/978-3-658-16502-4.

Basco, Ana Inés, Gustavo Beliz, Diego Coatz y Paula Garnero. 2018. Industria 4.0: fabricando un futuro. Washington D. C.: Banco Interamericano de Desarrollo. http://dx.doi.org/ $10.18235 / 0001229$.

Branger, Jakob, y Zhibo Pang. 2015. "From Automated Home to Sustainable, Healthy and Manufacturing Home: A New Story Enabled by the Internet-of-Things and Industry 4.0". Journal of Management Analytics 2 (4): 314-332. https://doi.org/10.1080/23270012.201 5.1115379 .

Calvo, Patrici. 2017. "Ética de las cosas ante el desafío de la Industria 4.0". Gobernanza 4 (12): 2-5. http://bit.ly/2uLO3Yd. 
Coyle, John, John Jr. Langley, Robert Novarck y Brian Gibson. 2018. Administración de la cadena de suministro, una perspectiva logística. Ciudad de Mexico: Limusa.

Crafts, Nicholas. 1996. "The First Industrial Revolution: A Guided Tour for Growth Economists". American Economic Review 86 (2): 197-201. http://bit.ly/323cOvi.

Cueva Lovelle, Juan Manuel, José Ignacio Rodríguez Molano y Carlos Enrique Montenegro Marín. 2015. "Introducción al internet de las cosas". Redes de Ingeniería 6: 51-59. https:// doi.org/10.14483/2248762X.8505.

Del Val Román, José Luis. 2016. "Industria 4.0: la transformación digital de la industria". Informe COODDI. 1-10. Accedido 23 de junio de 2020. https://bit.ly/2zDyDrk.

Dilberoglu, Ugur M., Bahar Gharehpapagh, Ulas Yaman y Melik Dolen. 2017. "The Role of Additive Manufacturing in the Era of Industry 4.0". Procedia Manufacturing 11: 545-554. https://doi.org/10.1016/j.promfg.2017.07.148.

Dopico, Miguel, Alberto Gómez, David de la Fuente, Nazario García, Rafael Rosillo y Julio Puche. 2016. "A Vision of Industry 4.0 from an Artificial Intelligence Point of View". Proceedings of the International Conference on Artificial Intelligence ICAI 16: 407-413. https://bit.ly/2AUP2s8.

Fernández, Miguel Ángel, y Roberto Pajares. 2016. "La digitalización del mundo industrial", Economía Industrial 405: 41-45. https://bit.ly/2Nm4bFu.

Frank, Alejandro Germán, Lucas Santos Dalenogare y Néstor Fabián Ayala. 2019. "Industry 4.0 Technologies: Implementation Patterns in Manufacturing Companies". International Journal of Production Economics (210): 15-26. https://doi.org/10.1016/j.ijpe.2019.01.004.

Gil, Xema. 2017. "Industria 4.0 apuesta por la sostenibilidad”. Accedido 23 de junio de 2020. http://bit.ly/2wdUwLL.

González Muntadas, Joaquim. 2016. "Industria 4.0, RSE, Robots y Personas". Diario Responsable. Accedido 23 de junio de 2020. http://bit.ly/3bEokkW.

Hidayat, Muhammad, y Ulani Yunus. 2019. "The Entrepreneurship Learning in Industrial 4.0 Era (Case Study in Indonesian College)”. Journal of Entrepreneurship Education 22 (5): 1-15. https://bit.ly/3fQTRS3.

Huang, Zishuo, Hang Yu, Zhenwei Peng y Yifu Feng. 2017. "Planning Community Energy System in the Industry 4.0 Era: Achievements, Challenges and a Potential Solution". Renewable and Sustainable Energy Reviews (78): 710-721. https://doi.org/10.1016/j.rser. 2017.04.004.

Humphries, Jane, y Benjamin Schneider. 2018. "Spinning the Industrial Revolution". The Economic History Review 71 (2): 455-460. https://doi.org/10.1111/ehr.12693.

Kagermann, Henning, Wolfgang Wahlster y Johannes Helbig. 2013. Recommendations for Implementing the Strategic Initiative Industry 4.0. Technical Report. Acatech. Accedido 4 de noviembre de 2019. http://bit.ly/37wzC7A.

Kolberg, Dennis, y Detlef Zühlke. 2015. "Lean Automation Enabled by Industry 4.0 Technologies". IFAC-PapersOnLine 48 (3): 1870-1875. https://doi.org/10.1016/j.ifacol.2015.06. 359. 
Kovar, Jiri, Katerina Mouralova, Filip Ksica, Jiri Kroupa, Ondrej Andrs y Zdenek Hadas. 2016. "Virtual Reality in Context of Industry 4.0 Proposed Projects at Brno University of Technology". Proceedings at 17th International Conference on Mechatronics Mechatronika, 1-7. https://bit.ly/2V7qIKm.

Malvicino, Facundo, y Gabriel Yoguel. 2016. "Big Data. Avances recientes a nivel internacional y perspectivas para el desarrollo local". Centro Interdisciplinario de Estudios en Ciencia, Tecnología e Innovación. Buenos Aires. Accedido diciembre de 2019. https://bit. ly/3hOKJiJ.

Mattern, Friedemann. 2013. "Die technische Basis für M2M und das Internet der Dinge". M2M und das Internet der Dinge - Vom Hype zur praktischen Nutzung. Editor: Prof. Dr. Jörg Eberspächer y Prof. Dr. Uwe Kubach. Munich. Münchner Kreis - Übernationale Vereinigung für Kommunikationsforschung e.V., 56-69. https://bit.ly/2Yt77qk.

Moeuf, Alexandre, Robert Pellerin, Samir Lamouri, Simón Tamayo-Giraldo y Rodolphe Barbaray. 2018. "The Industrial Management of SMEs in the Era of Industry 4.0". International Journal of Production Research 56 (3): 1118-1136. https://doi.org/10.1080/00207 543.2017.1372647.

Mokyr, Joel. 1998. The Second Industrial Revolution, 1870-1914. Evanston: Northwestern University. https://bit.ly/2Z5P7B9.

---. 1999. The British Industrial Revolution: An Economic Perspective. Nueva York. Westview Press. Boulder. https://doi.org/10.4324/9780429494567.

Mora, Dorian, Marco Taisch y Armando Walter Colombo. 2012a. "Energy Efficiency Software Tools: State-of-the-Art towards Sustainable Industrial Automation". Proceedings of the 10th GCSM Global Conference on Sustainable Manufacturing, Estambul, 31 de octubre a 2 de noviembre de 2012, 730-735. http://bit.ly/2Hoip5A.

---. 2012b. "Towards an Energy Management System of Systems: An Industrial Case Study". Proceedings of IECON 2012-38th Annual Conference on IEEE Industrial Electronics Society. Montreal, 25-28 de octubre de 2012, 5811-5816. https://doi.org/10.1109/IECON. 2012.6389588 .

Mora, Dorian, Marco Taisch, Armando Walter Colombo y Joao Marco Mendes. 2012. "Service-Oriented Architecture Approach for Industrial System of Systems: State-of-the-Art for Energy Management". Proceedings of IEEE 10th International Conference on Industrial Informatics INDIN 2012. Beijing, 25-27 de julio, 1246-1251. https://doi.org/ 10.1109/INDIN.2012.6301130.

Mora Sánchez, Dorian Oswaldo. 2019a. "Corporate Social Responsibility Challenges and Risks of Industry 4.0 technologies: A review". Proceedings of Smart SysTech 2019-European Conference on Smart Objects, Systems and Technologies. Magdeburg, 4-5 de junio de 2019. VDE, 1-8. https://bit.ly/2Yx7YX7.

---. 2019b. "Sustainable Development Challenges and Risks of Industry 4.0: A Literature Review". Proceedings of Global IoT Summit (GIoTS'19). Aarhus, 17-21 de junio de 2019, 1-6. https://doi.org/10.1109/GIOTS.2019.8766414. 
Mowery, David. 2009. "Plus Can Change: Industrial R\&D in the Third Industrial Revolution". Industrial and Corporate Change" 18 (1): 1-50. https://doi.org/10.1093/icc/dtn049.

Müller, Julian Marius, Oana Buliga y Kai-Ingo Voigt. 2018. "Fortune Favors the Prepared: How SMEs Approach Business Model Innovations in Industry 4.0”. Technological Forecasting and Social Change 132: 2-17. https://doi.org/10.1016/j.techfore.2017.12.019.

Murazzo, María Antonia, Nelson R. Rodríguez, Daniela A. Villafañe y Daniel Gallardo. 2013. "Desarrollo de Aplicaciones Colaborativas para Cloud Computing". Libro de Actas CACIC2013. Mar del Plata. Accedido 23 de junio de 2020. https://bit.ly/31atgex.

Neumann, Donald. 2019. Flotas 4.0. revista logística en foco. Río de Janeiro: MAG Editora.

Ortega Moody, Jorge Alberto, Roger Ernesto Sánchez Alonso, José-Joel González Barbosa y Guillermo Reyes Morales. 2016. "Virtual Laboratories for Training in Industrial Robotics”. IEEE Latin America Transactions 14 (2): 665-672. https://doi.org/10.1109/TLA. 2016.7437208 .

Paelke, Volker. 2014. "Augmented Reality in the Smart Factory: Supporting Workers in an Industry 4.0. Environment". Proceedings of the 2014 IEEE Emerging Technology and Factory Automation (ETFA). Barcelona, 16-19 de septiembre de 2014, 1-4. https://doi.org/ 10.1109/ETFA.2014.7005252.

Pieroni, Alessandra, Noemi Scarpato y Marco Brilli. 2018. "Industry 4.0 Revolution in Autonomous and Connected Vehicle a Non-conventional Approach to Manage Big Data". Journal of Theoretical and Applied Information Technology 96: 10-18. Accedido 23 de junio de 2020. https://bit.ly/3fTMEAQ.

Ramachandran Venkatapathy, Aswin Karthik, Andreas Riesner, Moritz Roidl, Jan Emmerich y Michael ten Hompel. 2015. "PhyNode: An Intelligent, Cyber-physical System with Energy Neutral Operation for PhyNetLab”. Proceedings of Smart SysTech 2015 - European Conference on Smart Objects, Systems and Technologies. Aachen, 16-17 de julio de 2015. https://bit.ly/3duJIci.

Ries, Marco S., y Geert Gins. 2017. "Industrial Process Monitoring in the Big Data/Industry 4.0 Era: from Detection, to Diagnosis, to Prognosis". Processes 5 (3): 34-42. https://doi. org/10.3390/pr5030035.

Rodič, Blaž. 2017. "Industry 4.0 and the New Simulation Modelling Paradigm". Orga 17 (20): 193-207. https://doi.org/10.1515/orga-2017-0017.

Rodríguez Murcia, Humberto. 2008. "Development of Solar Energy in Colombia and its Prospects”. Revista de Ingeniería 28: 83-89. https://bit.ly/2AC4JEF.

Salhaoui, Marouane, Mounir Ariouna, Antonio Guerrero-González y María Socorro García-Cascales. 2018. "An IoT Control System for Wind Power Generators. Information Processing and Management of Uncertainty in Knowledge-Based Systems Applications". IPMU 20 (12): 469-479. https://doi.org/10.1007/978-3-319-91479-4_39.

Sancho Hirare, Carolina. 2017. "Ciberseguridad". Revista Latinoamericana de Estudios de Seguridad 20: 78-89. http://bit.ly/38x3t1g.

Schwab, Klaus. 2017. The Fourth Industrial Revolution. Genova: Crown Publishing Group. 
Serna, Félix, Carlos Catalán y Alfonso Blesa. 2015. "Industria 4.0 en el grado de ingeniería electrónica y automática". Actas de las XXI Jornadas de la Enseñanza Universitaria de la Informática. Andorra la Vieja: 327-332. Accedido 23 de junio de 2020. https://bit. ly/3exUEax.

Shrouf, Fadi, Joaquín Ordieres y Giovanni Miragliotta. 2014. "Smart Factories in Industry 4.0: A Review of the Concept and of Energy Management Approached in Production based on the Internet of Things paradigm". IEEM 112: 679-701. https://doi.org/10.1109/ IEEM.2014.7058728.

Stock, Tim, Michael Obenaus, Sascha Kunz y Holger Kohl. 2018. "Industry 4.0 as Enabler for a Sustainable Development: A Qualitative Assessment of its Ecological and Social Potential”. Process Safety and Environmental Protection 118: 254-267. https://doi.org/10.1016/j.psep.2018.06.026.

Talwar, Shilpa, Debabani Choudhury, Konstantinos Dimou, Ehsan Aryafar, Boyd Bangerter y Kenneth Stewart. 2014. "Enabling Technologies and Architectures for 5G Wireless". 2014-IMS-5G (120): 54-69. https://doi.org/10.1109/MWSYM.2014.6848639.

Telukdarie, Arnesh, Eyad Buhulaiga, Surajit Bag, Shivam Gupta y Zongwei Luo. 2018. "Industry 4.0 Implementation for Multinationals". Process Safety and Environmental Protection 118: 316-329. https://doi.org/10.1016/j.psep.2018.06.030.

The Intergovernmental Panel on Climate Change (IPCC). 2011. Fuentes de energía renovables y mitigación del cambio climático. Resumen para responsables de políticas y resumen técnico. Ginebra: IPCC. https://bit.ly/2VaTSbN.

Thoben, Klaus-Dieter, Stefan Wiesner y Thorsten Wuest. 2017. "Industrie 4.0 and Smart Manufacturing: A Review of Research Issues and Application Examples". International Journal of Automation Technology 11 (1): 4-19. https://doi.org/10.20965/ijat.2017.p0004.

Thompson, Mary Kathryn, Giovanni Morini, Tom Vaneker, Georges Fadel, Ian Campbell, Ian Gibson, Alain Bernard, Joachim Schulz, Patricia Graf, Bhrigu Ahuja y Filomeno Martina. 2016. "Design for Additive Manufacturing: Trends, Opportunities, Considerations, and Constraints". CIRP Annals 65 (2): 737-760. https://doi.org/10.1016/j.cirp.2016.05.004.

Vachálek, Ján, Lukás Bartalský, Oliver Rovný, Dana Šišmišová, Martin Morháč y Milan Lokšík. 2017. "The Digital twin of an Industrial Production Line within the Industry 4.0 Concept”. 21st International Conference on Process Control (PC) 6: 234-239. https://doi. org/10.1109/PC.2017.7976223.

Van der Velden, Maja. 2018. "Digitalization and the UN Sustainable development Goals: What Role for Design”. Interaction Design and Architecture(s) Journal 37: 160-174. https://bit. ly/3hWxw7y.

Wang, Lihui, Martin Törngren y Mauro Onori. 2015. "Current Status and Advancement of Cyber-Physical Systems in Manufacturing”. Journal of Manufacturing Systems 37: 517527. https://doi.org/10.1016/j.jmsy.2015.04.008.

Wang, Shiyong, Jiafu Wan, Daqiang Zhang, Di Li y Chunhua Zhang. 2016. "Towards Smart Factory for Industry 4.0: a Self-organized Multi-Agent System with Big Data based Fee- 
dback and Coordination". Computer Networks 101: 158-168. https://doi.org/10.1016/j. comnet.2015.12.017.

Wang, Shiyong, Jiafu Wan, Di Li y Chunhua Zhang. 2016. "Implementing Smart Factory of Industrie 4.0: An Outlook". International Journal of Distributed Sensor Networks 12 (1): 58-64. https://doi.org/10.1155/2016/3159805.

Xu, Shaoyi, Yan Li, Yuan Gao, Yang Liu y Haris Gačanin. 2018. “Oportunistic Coexistence of LTE and WiFi for Future 5G System: Experimental Performance Evaluation and Analysis". IEEE Access 6: 8725-8741. https://doi.org/10.1109/ACCESS.2017.2787783.

Yin, Yong, Kathryn E. Stecke y Dongni Li. 2018. "The Evolution of Production Systems from Industry 2.0 Through Industry 4.0". International Journal of Production Research 56 (12): 848-861. https://doi.org/10.1080/00207543.2017.1403664.

Zhou, Jiehan, Jukka Riekki, Mätti Hämäläinen, Pasi Mattila, Xinguo Yu, Xiwei Liu y Weishan Zhang. 2018. "China-Finland EduCloud Platform Towards Innovative Education". Pacific-Rim Symposium on Image and Video Technology 5 (3): 172-185. https://doi. org/10.1007/978-3-319-92753-4_14. 